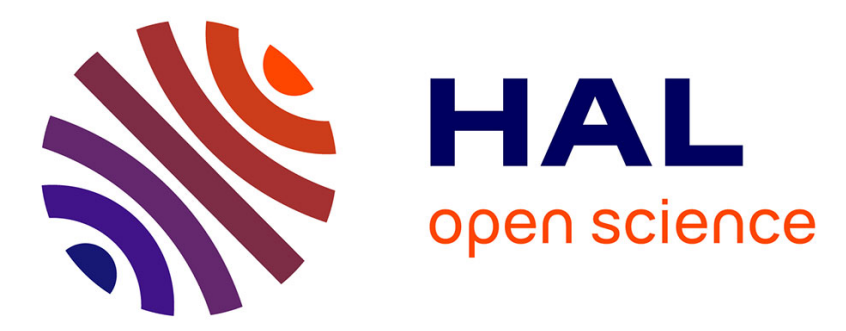

\title{
Coupled Parallel Snakes For Segmenting Healthy and Pathological Retinal Arteries in Adaptive Optics Images
}

Nicolas Lermé, Florence Rossant, Isabelle Bloch, Michel Paques, Edouard Koch

\section{- To cite this version:}

Nicolas Lermé, Florence Rossant, Isabelle Bloch, Michel Paques, Edouard Koch. Coupled Parallel Snakes For Segmenting Healthy and Pathological Retinal Arteries in Adaptive Optics Images. 2014. hal-01015307v2

\author{
HAL Id: hal-01015307 \\ https://hal.science/hal-01015307v2
}

Preprint submitted on 6 Jul 2014

HAL is a multi-disciplinary open access archive for the deposit and dissemination of scientific research documents, whether they are published or not. The documents may come from teaching and research institutions in France or abroad, or from public or private research centers.
L'archive ouverte pluridisciplinaire HAL, est destinée au dépôt et à la diffusion de documents scientifiques de niveau recherche, publiés ou non, émanant des établissements d'enseignement et de recherche français ou étrangers, des laboratoires publics ou privés. 


\title{
Coupled Parallel Snakes For Segmenting Healthy and Pathological Retinal Arteries in Adaptive Optics Images
}

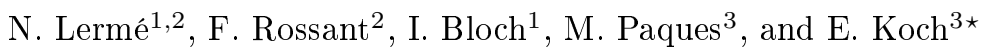 \\ 1 Institut Mines-Télécom, Télécom ParisTech, CNRS LTCI, Paris, France \\ ${ }^{2}$ LISITE, Institut Supérieur d'Électronique de Paris, Paris, France \\ 3 CIC 503, Centre Hospitalier National des Quinze-Vingts, Paris, France
}

\begin{abstract}
In this paper, we propose two important improvements of an existing approach for automatically segmenting the walls of retinal arteries of healthy / pathological subjects in adaptive optics images. We illustrate the limits of the previous approach and propose to (i) modify the pre-segmentation step, and (ii) embed additional information through coupling energy terms in the parallel active contour model. The interest of these new elements as well as the pre-segmentation step is then evaluated against manual segmentations. They improve the robustness against low contrasted walls and morphological deformations that occur along vessels in case of pathologies. Noticeably, this strategy permits to obtain a mean error of $13.4 \%$ compared to an inter-physicians error of $17 \%$, for the wall thickness which is the most sensitive measure used. Additionally, this mean error is in the same range than for healthy subjects.
\end{abstract}

Keywords: Active contour model, adaptive optics, retina imaging.

\section{Introduction}

Arterial hypertension and diabetes mainly and precociously affect the physiology and the structure of retinal blood vessels of small diameter (i.e. less than $150 \mu \mathrm{m})$. According to the Public Health Agency of Canada, these chronic diseases affected 15 to $20 \%$ of the world's adult population in 2009. Hypertensive retinopathy (HR) and diabetic retinopathy (DR) are common ocular complications of the above diseases. The lesions caused by these complications include diffuse or focal narrowing, or dilation of the vessel and of the wall. Although HR and DR do not present early warning signs, they are predictive of endorgan damage such as stroke or visual loss [1,4]. In [8], the authors estimate that $98 \%$ of visual damages could be avoided if DR was treated in time. Accurate measurements of walls are therefore necessary to better prevent the DR and the complications of the HR. Fundus photographs and Doppler-based measurements cannot however resolve the vessel walls due to their limited spatial resolution. Adaptive Optics (AO) is a recent opto-electronic technology that improves the

\footnotetext{
^ This work is funded by the ANR project ANR-12-TECS-0015-03 (2013-2015).
} 


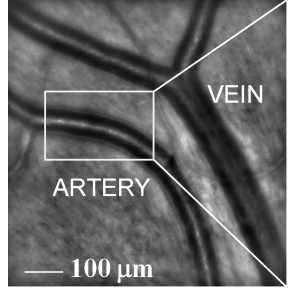

(a) $924 \times 947$

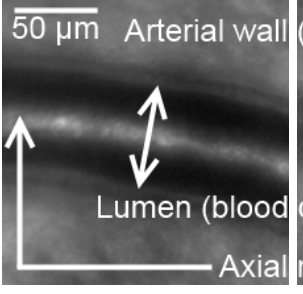

(b) $218 \times 218$

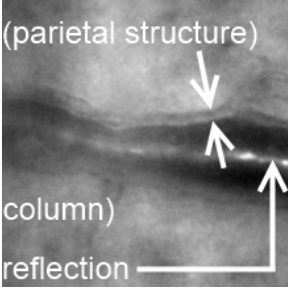

(c) $218 \times 218$

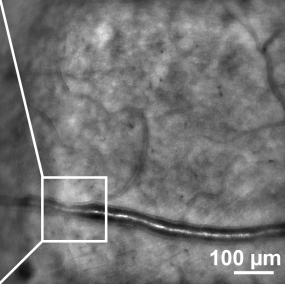

(d) $900 \times 900$

Fig. 1: Examples of images acquired by the $\mathrm{AO}$ camera [9] and a detailed view of them for an healthy subject (a),(b) and a pathological one (c),(d). The sizes below each image are expressed in pixels.

resolution of fundus photographs. AO-based cameras permit to visualize microstructures such as vascular walls [2], noninvasively. In the present study, the rtx 1 camera [9] is used to acquire 2D images by flood illumination at $10 \mathrm{~Hz}$ using a $850 \mathrm{~nm}$ LED light source with a pixel-resolution of $0.8 \mu \mathrm{m}$. These images are registered and averaged to increase the signal-to-noise ratio [6]. In the resulting images, blood vessels appear as dark elongated structures with a bright linear axial reflection, over a textured background. Outer borders of walls are however only visible along arteries and the present study will focus on them. Parietal structures (arterial walls) appear as a gray line along both sides of the lumen (blood column), with a thickness of about $15 \%$ of it [5] (see Figure 1).

Segmenting the artery walls in these images is a challenging problem. For both pathological and healthy subjects, (i) the background of the related images is highly textured, (ii) significant intensity changes can occur along axial reflections, (iii) the outer borders of walls are low contrasted, and (iv) some vessel segments can be locally blurred due to the geometry of the retina. This problem is by far more challenging for images from pathological cases since a large variability of morphological deformations can locally occur along arteries, which generally present a poor contrast on walls. Since AO-based fundus cameras remain uncommon yet, only few papers have addressed this issue. Recently, an automatic procedure was proposed where the walls are modeled as four curves approximately parallel to a common reference line located near axial reflections [7]. Once the line is detected, the artery walls are pre-segmented using a tracking procedure to cope with morphological deformations. Then, they are segmented using an active contour model embedding a parallelism constraint to both control their distance to the reference line and improve the robustness against the noise and lack of contrast. The accuracy of this approach on pathological cases has however not been assessed.

In this paper, we propose two important improvements to this approach: (i) a piecewise constant estimation of the vessel diameter in the first stage of the presegmentation, and (ii) coupled energy terms in the active contours model. The purpose of these new elements is to improve the robustness against noise, low 
contrasted walls and morphological deformations in case of pathologies, while keeping a computational cost similar to the one of [7].

The rest of this paper is organized as follows. In Section 2, we briefly remind the approach in [7] for segmenting arterial walls on healthy subjects. Afterwards, we illustrate the limits of this approach and present the above new elements, in Section 3. Finally, we evaluate in Section 4 the relevance of the pre-segmentation step as well as these new elements, against manual segmentations.

\section{An approach for segmenting arterial walls and its limits}

\subsection{Description}

In this section, we remind the strategy presented in [7] for segmenting walls of retinal arteries. Let us consider one artery branch represented by a fixed curve located on the axial reflection, resulting of an automatic segmentation process [7]. We denote this branch as the reference line $V(s)=(x(s), y(s))^{T}$, parameterized by $s$. The artery walls are modeled by $V_{1}, V_{2}$ (inner) and $V_{3}, V_{4}$ (outer), four curves approximately parallel to the reference line $V$, defined by:

$$
\left\{\begin{array} { l } 
{ V _ { 1 } ( s ) = V ( s ) + b _ { 1 } ( s ) \boldsymbol { n } ( s ) } \\
{ V _ { 2 } ( s ) = V ( s ) - b _ { 2 } ( s ) \boldsymbol { n } ( s ) } \\
{ V _ { 3 } ( s ) = V ( s ) + b _ { 3 } ( s ) \boldsymbol { n } ( s ) } \\
{ V _ { 4 } ( s ) = V ( s ) - b _ { 4 } ( s ) \boldsymbol { n } ( s ) }
\end{array} \quad \text { such that } \quad \left\{\begin{array}{l}
b_{1}(s)<b_{3}(s) \\
b_{2}(s)<b_{4}(s),
\end{array} \quad \forall s\right.\right.
$$

where $\boldsymbol{n}(s)$ is the normal vector to $V$ and $b_{k}(s)$ is the local distance (or halfdiameter) of any curve $V_{k}$ to $V$. In the above model, notice the direct correspondence between the points of any curve $V_{k}$ to those of the reference line $V$. The segmentation procedure amounts to compute the half-diameters $\left\{b_{k}\right\}_{k=1, \ldots, 4}$.

The artery walls are first pre-segmented. This is achieved by simultaneously positioning the curves using a tracking procedure to cope with morphological deformations. All along this pre-segmentation, it is assumed that the opposite curves lie at the same distance from the reference line $V$ (i.e. $b_{1}(s)=b_{2}(s)=$ $b^{\text {int }}(s)$ and $\left.b_{3}(s)=b_{4}(s)=b^{\text {ext }}(s), \forall s\right)$ and that the wall thickness is constant (i.e. $\left.b^{e x t}(s)-b^{\text {int }}(s)=c s t, \forall s\right)$. The reference line $V$ and all the elements that refer to it are discretized into $m$ equally spaced points. The pre-segmentation is based on a criterion to be maximized, defined for every $i \in\{1, \ldots, m\}$ by:

$$
G\left(b^{i}, b^{e}, i\right)=\frac{\bar{D}^{i n t}\left(b^{i}, i, r\right)}{\bar{I}^{i n t}\left(b^{i}, i, r\right)}+\bar{D}^{\text {ext }}\left(b^{e}, i, r\right),
$$

where $\bar{D}^{i n t}$ and $\bar{I}^{i n t}$ respectively denote the mean local gradient and the mean local intensity along inner curves while $\bar{D}^{\text {ext }}$ denote the mean local gradient along outer curves. $\bar{D}^{\text {int }}, \bar{I}^{\text {int }}$ and $\bar{D}^{\text {ext }}$ are averaged over a small window of size $(2 r+1)$. The function $G$ encourages inner and outer curves to be located near large image gradients. Dividing by the mean intensity also favors inner curves to be located in dark areas. The pre-segmentation aims at estimating inner and outer half-diameters $b^{\text {int }}(s), b^{\text {ext }}(s)$ and consists of the following steps: 
1. Constant half-diameters $b^{\text {int }}{ }_{0}$ and $b^{e x t_{0}}$ along the artery, are estimated. This is achieved by maximizing the mean of $G\left(b^{i}, b^{e}, i\right)$ over $\left(b^{i}, b^{e}\right) \in\left(\mathbb{R}^{+} \times \mathbb{R}^{+}\right)$ along the curves defined by the half-diameters $b^{i}$ and $b^{e}$. We then denote by $\bar{e}=\left(b^{e x t_{0}}-b^{i n t_{0}}\right)$ the wall thickness estimated from the resulting curves.

2. The position $i^{*}$ maximizing (2) along the curves from Step 1 is determined.

3. Variable half-diameters $b^{\text {int }}(s)$ and $b^{\text {ext }}(s)=b^{\text {int }}(s)+\bar{e}+e$ are estimated using tracking by maximizing for any $i \in\left\{i^{*}+1, \ldots, m\right\}$ and for $j=i-1$ :

$$
\alpha G\left(b^{i n t}(i), b^{i n t}(i)+\bar{e}+e, i\right)-(1-\alpha)\left(b^{i n t}(i)-b^{i n t}(j)\right)^{2}, \quad \alpha \in[0,1],
$$

where $\alpha$ is a regularization parameter. The closer $\alpha$ is to zero, the more the right term in (3) penalizes large local deviations of $b^{\text {int }}$. The above scheme is also applied to any $i \in\left\{i^{*}-1, \ldots, 1\right\}$ and for $j=i+1$. Once $b^{\text {int }}$ has been fixed for any error $e$, the optimal error $e^{*}$ is taken as the one that maximizes the sum of (3) over any $i \in\{2, \ldots, m\}$ and for $j=i-1$ (see [7] for details).

Then, an active contour model (parallel snakes) is applied to accurately position the curves found by the pre-segmentation. This model is an extension of [3] for extracting four curves approximately parallel to a fixed reference line $V$. This is achieved by minimizing

$$
E\left(V_{1}, \ldots, V_{4}, b_{1}, \ldots, b_{4}\right)=\sum_{k=1}^{4}(\underbrace{\int_{0}^{1} P\left(V_{k}(s)\right) d s}_{E_{\text {Image }}\left(V_{k}\right)}+\underbrace{\int_{0}^{1} \varphi_{k}(s)\left(b_{k}^{\prime}(s)\right)^{2} d s}_{R\left(V_{k}, b_{k}\right)}),
$$

where $E_{\text {Image }}$ is designed to attract the curves towards large image gradients while $R$ controls the variation of the half-diameter $b_{k}(s)$, thus imposing a local parallelism. The strength of this parallelism is controlled by the parameters $\left\{\varphi_{k}\right\}_{k=1, \ldots, 4}$ : the larger these parameters are, the more strict the parallelism to the reference line $V$ is. It is worth noting that the assumptions made for the presegmentation are fully relaxed, i.e. the four curves can now evolve independently of each other. They are implicitly linked by the parallelism constraint but no symmetry property w.r.t. the reference line $V$ is imposed. The minimization of (4) is obtained by solving the Euler-Lagrange equations w.r.t. the half-diameters $b_{k}$. The resulting algorithm uses standard numerical approximation of derivatives and converges until some accuracy $\varepsilon$ is reached.

\section{$2.2 \quad$ Limits}

The approach detailed in [7] has two important limitations. For some cases, the pre-segmentation can fail to accurately position the curves close to artery walls. This situation is illustrated in the top row of Figure 2. The inner and outer halfdiameters are underestimated at Step 1 due to the local narrowing of the artery. These poor estimations therefore prevent to perform the tracking procedure from a good initial position found at Step 2. The tracking procedure therefore fails to follow correctly morphological deformations. This suggests a more robust way to estimate the half-diameters at Step 1. 
For some cases, the parallel snake model can also fail to accurately position the curves close to artery walls. This situation is illustrated in the bottom row of Figure 2. Although the solution provided by the pre-segmentation is correct, the positioning of the curves is inconsistent on an artery segment. Such a behavior is due to a poor contrast on the outer borders of walls. This suggests to enforce the regularity of the wall thickness along the arteries and also symmetry properties w.r.t. the reference line, as wall thicknesses are generally similar on both sides. Last but not least: the model (4) does not ensure that the constraints expressed in (1) hold. This point must also be addressed to ensure the anatomical consistency of the solution.

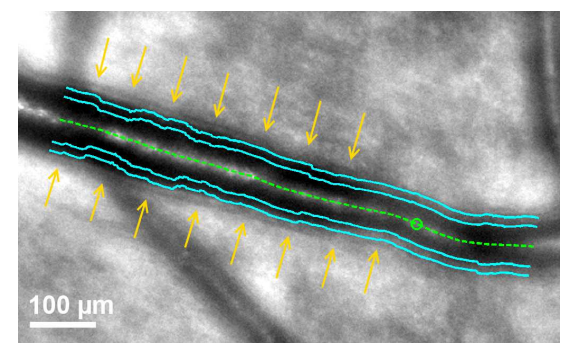

(a) $1027 \times 627$

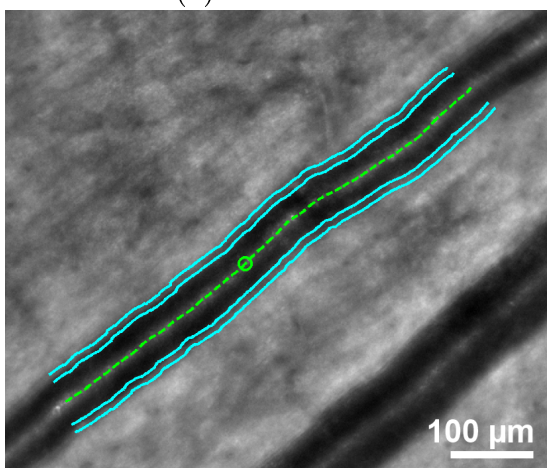

(c) $818 \times 692$

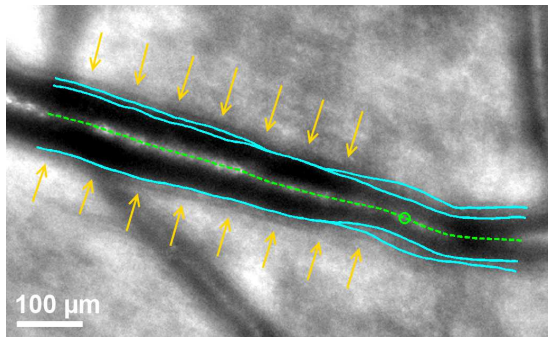

(b) $1027 \times 627$

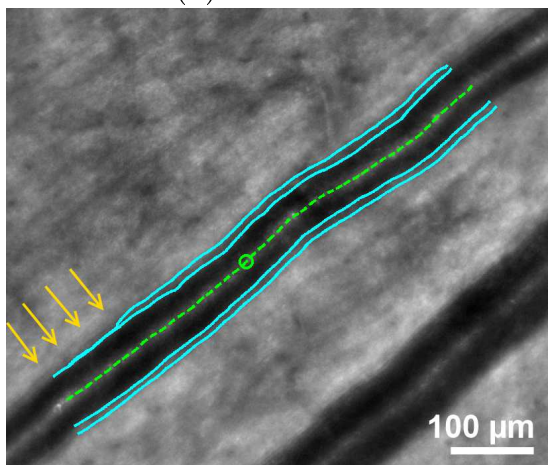

(d) $818 \times 692$

Fig. 2: Limits of the pre-segmentation (a,b) and the parallel snake model (c,d) for two distinct pathological subjects. Pre-segmentations and segmentations are respectively given in $(a, c)$ and $(b, d)$ columns, superimposed on the original image. Yellow arrows point misplacements of curves. The green dashed line corresponds to the reference line while the circle denotes the position from which the tracking procedure operates (see Step 2). The size of the images are expressed in pixels.

\section{Improvements}

\subsection{Pre-segmentation}

We propose a simple modification to overcome the difficulties outlined in Section 2.2. Instead of estimating inner and outer half-diameters as constant along 
vessels in Step 1, we propose to estimate them as piecewise constant. Then, the initial estimates of the inner and outer half-diameters are those found at the position maximizing (2) along the resulting piecewise constant curves (Step 2). In such a manner, these new half-diameters estimates are less prone to morphological deformations while keeping a good robustness against intensity changes along the artery walls. The robustness of the tracking process (Step 3) is therefore improved. In accordance to morphometric features, the piecewise constant estimations are performed on vessel segments whose length is 50 pixels $(\simeq 40 \mu \mathrm{m})$.

\subsection{Coupled Parallel Snakes model}

To overcome the difficulties of the parallel snake model highlighted in Section 2.2, we propose to modify the energy (4) (see Section 2.1) as follows

$$
\begin{aligned}
E\left(V_{1}, \ldots, V_{4}, b_{1}, \ldots, b_{4}\right)= & \sum_{k=1}^{4}\left(E_{\text {Image }}\left(V_{k}\right)+R\left(V, V_{k}\right)\right)+S\left(V_{1}, V_{3}, b_{1}, b_{3}\right) \\
& +S\left(V_{2}, V_{4}, b_{2}, b_{4}\right)+T\left(V_{1}, \ldots, V_{4}, b_{1}, \ldots, b_{4}\right),
\end{aligned}
$$

where

$$
\begin{gathered}
S\left(V_{i}, V_{j}, b_{i}, b_{j}\right)=\int_{0}^{1} \psi_{i, j}(s)\left(b_{j}(s)-b_{i}(s)-\beta_{i, j}^{0}\right)\left(b_{j}(s)-b_{i}(s)-\beta_{i, j}^{1}\right) d s, \\
T\left(V_{1}, \ldots, V_{4}, b_{1}, \ldots, b_{4}\right)=\int_{0}^{1} \lambda(s)\left(b_{3}(s)-b_{1}(s)-b_{4}(s)+b_{2}(s)\right)^{2} d s,
\end{gathered}
$$

and $\lambda, \psi_{1,3}, \psi_{2,4}$ are weighting parameters (independent of $s$ in our application). Notice that $E_{\text {Image }}$ and $R$ in (5) are the same as in (4). The term $T$ controls the wall thickness difference between $b_{3}(s)-b_{1}(s)$ and $b_{4}(s)-b_{2}(s), \forall s$. It reaches a minimum for $b_{3}(s)-b_{1}(s)=b_{4}(s)-b_{2}(s), \forall s$. The larger the parameter $\lambda$ is, the more identical the wall thickness on both sides of the reference line $V(s)$ is. The main role of $S$ is to control the variation of the wall thicknesses $b_{3}(s)-b_{1}(s)$ and $b_{4}(s)-b_{2}(s)$, with respect to the initial estimate $\left(b^{e x t}(s)-b^{i n t}(s)\right)$ found in the pre-segmentation step. This term reaches a minimum when the wall thickness is between $\beta_{i, j}^{0}$ and $\beta_{i, j}^{1}$, for $(i, j)=(1,3)$ or $(i, j)=(2,4)$. The larger the weighting parameters $\psi_{1,3}$ and $\psi_{2,4}$ are, the closer the wall thicknesses $b_{3}(s)-b_{1}(s)$ and $b_{4}(s)-b_{2}(s)$ are to $\left(b^{\text {ext }}(s)-b^{\text {int }}(s)\right)$. Here, we set for any $s$

$\beta_{1,3}^{0}=\beta_{2,4}^{0}=(1-\gamma)\left(b^{\text {ext }}(s)-b^{\text {int }}(s)\right), \quad \beta_{1,3}^{1}=\beta_{2,4}^{1}=(1+\gamma)\left(b^{e x t}(s)-b^{\text {int }}(s)\right)$,

where $\gamma \in[0,1]$. Since the resulting curves found by the pre-segmentation lie at the same distance from the reference line $V, S$ also controls both the amount of symmetry of the curves $V_{1} / V_{2}$ and $V_{3} / V_{4}$ w.r.t the reference line $V$. The larger the parameters $\psi_{1,3}$ and $\psi_{2,4}$ are, the more strict the symmetry is. Unlike (4), (5) ensures that the constraints (1) hold as the parameters $\psi_{1,3}$ and $\psi_{2,4}$ tend to $+\infty$. Notice that when the weighting parameters $\lambda, \psi_{1,3}$ and $\psi_{2,4}$ are null, the energy (5) becomes the same as (4). Finally, the energy (5) is minimized in the same way as for (4). 


\section{Experimental results}

\subsection{Data and experimental protocol}

17 images from pathological subjects were manually segmented by 3 physicians. These physicians have several years of experience in AO image interpretation. Each physician segmented the images ${ }^{4}$ two times, separated by several weeks to diminish the memory effect between both segmentations. The images were selected by the medical experts to ensure the representativeness of the quality of the images and the vessel deformations encountered during clinical routine.

Let us denote by $S_{1}$ and $S_{2}$ two distinct segmentations. To evaluate the accuracy of the segmentation $S_{1}$ w.r.t. the segmentation $S_{2}$, we choose to use the same measures as the ones described in [7], i.e. the absolute relative difference on the inner diameter $\delta_{i n t}$, the outer diameter $\delta_{\text {ext }}$ and the total walls thickness $\delta_{w t}$ (i.e. the difference between outer and inner diameters). For each image, notice that these measures are expressed in percentages and are performed on the intersection of all manual segmentations from medical experts for that image. Then, we first estimate the intra-physician variability by computing the mean and standard deviation of the above measures between the segmentations of the same image, delineated by the same physician (see Table 1). Table 1 provides the same statistics but for a unit displacement of one pixel all along a curve.

As shown in Table 1, the physician $\mathrm{Phys}_{3}$ has the smallest intra-physician variability for two measures out of three. Because this physician produced the most stable segmentations, we choose him as a reference and denote him as Phys Ref. Next, we pre-segment all images with either variable (VHDI) or constant (CHDI) half-diameter estimations and using $r=10$ (see Section 2.1). For both pre-segmentations, half-diameters are estimated in a piecewise constant manner, as proposed in Section 3.1. VHDI is for $\alpha=0.95$ while CHDI is for $\alpha=0$. From these pre-segmentations, parallel snakes (PS) and the coupled parallel snakes (CPS) models are applied (see Section 3.2). For both, we set $\varepsilon=0.05$ and $\varphi_{k}=100, \forall k \in\{1, \ldots, 4\}$. CPS is for $\gamma=0.5$ while PS is for $\psi_{1,3}=0$, $\psi_{2,4}=0$ and $\lambda=0$. The weighting parameters $\psi_{1,3}, \psi_{2,4}$ and $\lambda$ involved in the CPS model have been optimized experimentally on a training set ${ }^{5}$, consisting of eight images extracted from the database. The optimized values are the ones that minimize the mean of the overall errors $\delta_{\text {int }}$ and $\delta_{\text {ext }}$. A test set is composed of the nine remaining images. The optimized values are also applied in all configurations that involve the CPS, in both training and test sets. This leads to six distinct configurations: CHDI, VHDI, CHDI $+\mathrm{PS}, \mathrm{CHDI}+\mathrm{CPS}, \mathrm{VHDI}+\mathrm{PS}$ and VHDI + CPS. The accuracy of all of them is evaluated by computing the mean and the standard deviation of $\delta_{i n t}, \delta_{e x t}$ and $\delta_{w t}$ w.r.t. the manual segmentations from the physician Phys Ref. The results are summarized in Table 2 and illustrated in Figure 3. The inter-physicians error is also given in Table 2: it is estimated between the segmentations from the physicians Phys 1 and Phys 2 w.r.t. those from the physician Phys Ref.

\footnotetext{
${ }^{4}$ Each segmentation consists of a single artery branch.

${ }^{5}$ By convenience, we make the weighting parameters $\psi_{1,3}$ and $\psi_{2,4}$ equal.
} 


\subsection{Discussion}

First, the overall mean error on $\delta_{w t}$ is much larger than the overall mean errors on $\delta_{i n t}$ and $\delta_{e x t}$. Due to the size of the parietal structures, the measure $\delta_{w t}$ is indeed very sensitive to curves displacements. In Table 2, the accuracy for the CPS model is almost the same between the training and test databases, meaning a good generalization of the parameters $\psi_{1,3}, \psi_{2,4}$ and $\lambda$.

The accuracy of CHDI is poor w.r.t. the inter-physician error, significantly worse that the one obtained with VHDI. Although this accuracy is globally improved when applying the PS or CPS models (CHDI+PS or CHDI +CPS), the final results are always worse than when relying on variable half-diameters (VHDI), showing the importance of the tracking in the pre-segmentation.

As mentioned previously, the accuracy provided by VHDI is much closer to the inter-physician error than with CHDI. It is still improved with the application of the PS model, except for $\delta_{i n t}$ on the test database, and, in average, it is always improved after the application of the CPS model. The final mean errors (VHDI + PS or VHDI + CPS) are close to the inter-physician errors for the internal and external diameters and lower for the wall thickness measures, the most sensitive ones. Overall, the VHDI + CPS flow reaches the best accuracy.

These observations are confirmed by the results depicted in Figure 3. For both subjects, VHDI + CPS reaches globally a better accuracy for two measures out of three. The interest of using variable half-diameters against constant ones in the pre-segmentation step is clear. However, it is less obvious when comparing CPS against PS. For Subject 9, VHDI+CPS shows a better accuracy than VHDI+PS for $\delta_{i n t}$ and $\delta_{w t}$. The coupling permitted indeed to correct the position of the top inner curve. For Subject 7, the segmentation for VHDI+CPS is however a little worse than for VHDI+PS. This reduced performance is due to a local asymmetry w.r.t. the axial reflection. However, such asymmetries are very uncommon in practice (including on the presented images). Also, when we evaluate the interest of using either the energy terms $S$ or $T$ (see (5)), we found that the latter has a stronger impact on the accuracy than the former. Finally, we applied the VHDI + CPS flow on the database of healthy subjects (the one used in the study presented in [7]) and found an accuracy very close to the presented results, for all measures. Finally, for a typical $600 \mu \mathrm{m}$ long vessel branch, the processing time for the pre-segmentation and the CPS model is respectively about 120 and 30 secs. Future work will focus on a methodology for optimizing the parameterization of the CPS model (i.e. the weighting parameters $\psi_{1,3}, \psi_{2,4}$ and $\lambda$ ), according to the type of image to be segmented, in order to better manage the uncommon cases.

\section{References}

1. N.H. Buus, O.N. Mathiassen, M. Fenger-Grøn, M.N. Præstholm, I. Sihm, N.K. Thybo, A.P. Schroeder, K. Thygesen, C. Aalkjær, O.L. Pedersen, M.J. Mulvany, and K.L. Christensen. Small artery structure during antihypertensive therapy is an 

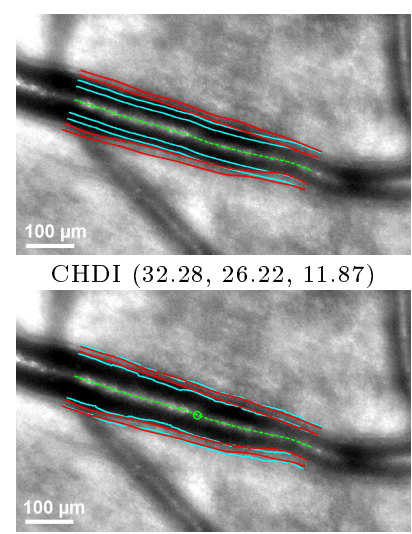

VHDI $(8.32, \underline{4.83}, 39.80)$

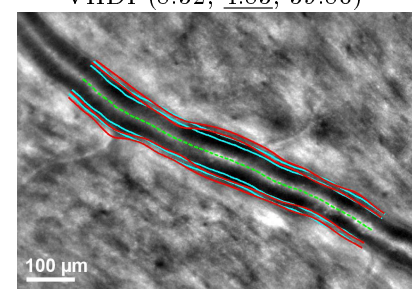

CHDI $(12.75,10.14,13.48)$

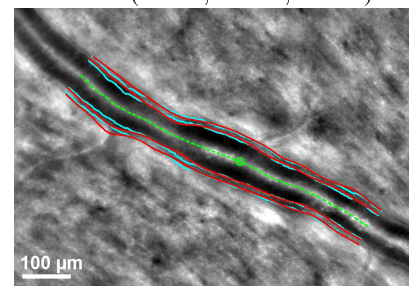

VHDI $(7.70,4.85,10.53)$

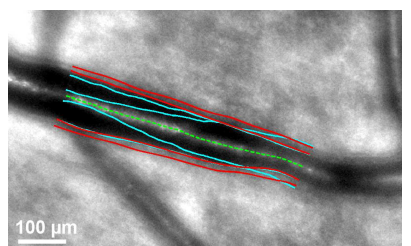

CHDI+PS $(35.26,22.57,87.50)$

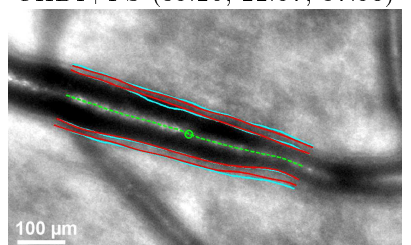

VHDI + PS $(2.75,5.44,25.44)$

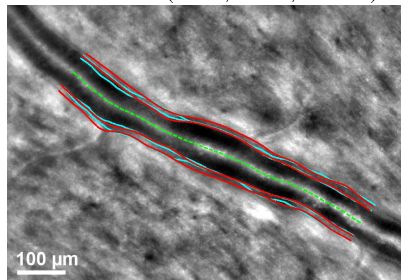

CHDI+PS $(5.56,10.15,37.94)$

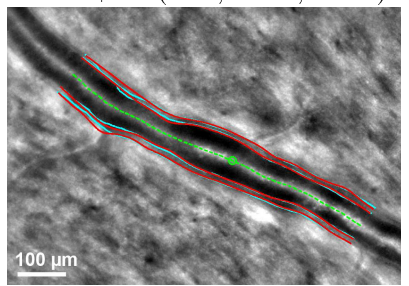

VHDI+PS $(4.36,4.01,16.10)$
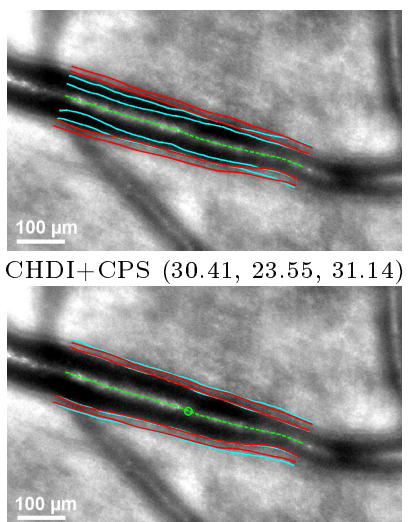

VHDI+CPS $(1.70,5.85, \underline{25.08})$

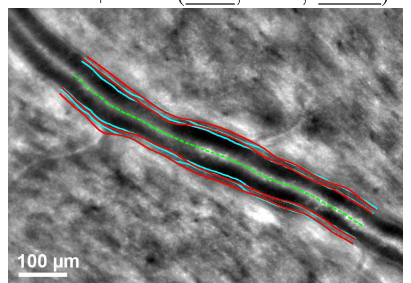

CHDI+CPS $(7.82,8.30,21.49)$

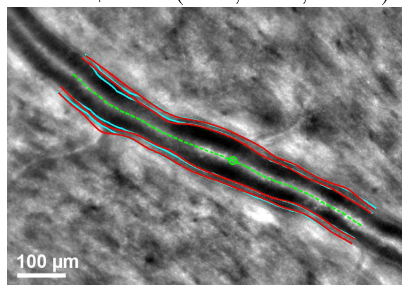

VHDI+CPS $(5.83, \underline{3.97}, \underline{10.36})$

Fig. 3: Results for Subject 9 (half-top) and 7 (half-down) against manual segmentations from the physician Phys Ref. Automatic and manual segmentations are respectively drawn in cyan and red. The green dashed line is the reference line. The green circles denote the position from which the tracking operates. The numbers below each image are respectively for $\delta_{i n t}, \delta_{\text {ext }}$ and $\delta_{w t}$ (in percentages). For each subject and each measure, the underlined numbers represent the configuration having the better accuracy among all the other ones. 


\begin{tabular}{|c|c|c|c|}
\hline & Phys $_{1} /$ Phys $_{1}$ & Phys $_{2} /$ Phys $_{2}$ & Phys $_{3} /$ Phys $_{3}$ \\
\hline ID & $4.0 \pm 3.1(1.0 \pm 0.3)$ & $4.7 \pm 3.8(1.0 \pm 0.3)$ & $4.3 \pm 3.8(1.1 \pm 0.3)$ \\
\hline OD & $3.3 \pm 2.8(0.8 \pm 2.2)$ & $4.1 \pm 3.5(0.8 \pm 0.2)$ & $2.6 \pm 2.1(0.8 \pm 0.2)$ \\
\hline WT & $16.5 \pm 15.6(2.9 \pm 0.9)$ & $23.8 \pm 26.4(3.4 \pm 1.3)$ & $15.2 \pm 12.3(2.9 \pm 0.8)$ \\
\hline
\end{tabular}

Table 1: Overall intra-physician variability for inner diameter (ID), outer diameter (OD) and wall thickness (WT). All measures are in percentages. The numbers in parentheses correspond to a unit displacement all along a curve.

\begin{tabular}{|c||c|c|c||c|c|c|c|}
\hline & CHDI & CHDI+PS & CHDI+CPS & VHDI & VHDI+PS & VHDI+CPS & Inter-physicians. \\
\hline ID & $8.8 \pm 7.3$ & $6.6 \pm 6.8$ & $6.7 \pm 6.8$ & $6.0 \pm 4.8$ & $5.8 \pm 4.3$ & $5.8 \pm 4.5$ & $4.5 \pm 3.7$ \\
\hline OD & $8.2 \pm 6.9$ & $8.2 \pm 8.1$ & $7.6 \pm 7.7$ & $4.6 \pm 4.3$ & $3.4 \pm 3.1$ & $3.3 \pm 3.0$ & $3.7 \pm 2.9$ \\
\hline WT & $19.5 \pm 16.2$ & $32.3 \pm 33.7$ & $26.2 \pm 27.7$ & $16.3 \pm 13.9$ & $15.5 \pm 12.7$ & $13.8 \pm 10.5$ & $18.1 \pm 14.1$ \\
\hline \hline ID & $11.2 \pm 12.6$ & $12.5 \pm 17.3$ & $11.0 \pm 13.6$ & $5.7 \pm 4.8$ & $6.3 \pm 6.4$ & $5.7 \pm 5.2$ & $4.9 \pm 2.2$ \\
\hline OD & $9.1 \pm 9.5$ & $8.1 \pm 9.2$ & $8.4 \pm 9.3$ & $4.2 \pm 3.2$ & $3.8 \pm 2.8$ & $3.9 \pm 2.8$ & $3.8 \pm 3.1$ \\
\hline WT & $18.6 \pm 17.0$ & $30.8 \pm 41.2$ & $21.2 \pm 20.7$ & $16.6 \pm 14.5$ & $14.6 \pm 12.9$ & $13.4 \pm 10.6$ & $17.0 \pm 13.1$ \\
\hline
\end{tabular}

Table 2: Accuracy of pre-segmentations with variable (VHDI) and constant (CHDI) half-diameters, parallel snakes (PS) and coupled parallel snakes (CPS), against manual segmentations from Phys $s_{\text {Ref }}$ for inner diameter (ID), outer diameter (OD) and walls thickness (WT). The three first and last rows are respectively for training and test sets. Mean and standard deviations are given in percentages. The mean error produced by a unit displacement of a curve for $\delta_{\text {int }}$, $\delta_{e x t}$ and $\delta_{w t}$ over the whole database is respectively $1.01 \%, 0.74 \%$ and $3.1 \%$.

independent predictor of cardiovascular events in essential hypertension. Journal of Hypertension, 31(4):791-797, 2013.

2. T.Y. Chan, D.A. Vannasdale, and S.A. Burns. The use of forward scatter to improve retinal vascular imaging with an adaptive optics scanning laser ophthalmoscope. Biomedical Optics Express, 3(10):2537-2549, 2012.

3. I. Ghorbel, F. Rossant, I. Bloch, and M. Paques. Modeling a parallelism constraint in active contours. Application to the segmentation of eye vessels and retinal layers. In International Conference on Image Processing (ICIP), pages 445-448, 2011.

4. A.M. Heagerty, C. Aalkjær, S.J. Bund, N. Korsgaard, and M.J. Mulvany. Small artery structure in hypertension: Dual processes of remodeling and growth. Journal of Hypertension, 21:391-397, 1993.

5. E. Koch. Morphometric study of human retina arteriolars in high resolution imaging. Master's thesis, Université Pierre et Marie Curie, 2012.

6. C. Kulcsár, G. Le Besnerais, E. Ödlund, and X. Levecq. Robust processing of images sequences produced by an adaptive optics retinal camera. In Optical Society of America, Adaptive Optics: Methods, Analysis and Applications, page OW3A.3, 2013.

7. N. Lermé, F. Rossant, I. Bloch, M. Paques, and E. Koch. Segmentation of retinal arteries in adaptive optics images. In International Conference on Pattern Recognition, 2014.

8. H. Taylor and J. Keeffe. World blindness: A 21st century perspective. British Journal of Ophtalmology, 85(3):261-266, 2001.

9. C. Viard, K. Nakashima, B. Lamory, M. Paques, X. Levecq, and N. Château. Imaging microscopic structures in pathological retinas using a flood-illumination adaptive optics retinal camera. In Photonics West: Biomedical Optics (BiOS), volume 7885, pages $788509+, 2011$. 